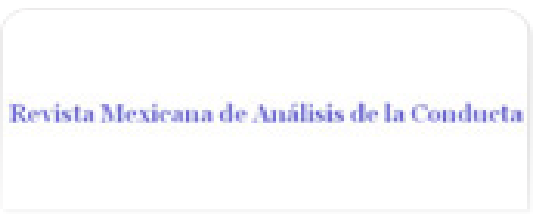

Revista Mexicana de Análisis de la Conducta ISSN: 0185-4534

editora@rmac-mx.org

Sociedad Mexicana de Análisis de la Conducta

México

FLORES-PINEDA, NELLY; VELÁZQUEZ-JURADO, HÉCTOR; SÁNCHEZ-SOSA, JUAN JOSÉ ESTRÉS, CONDUCTA DE AFRONTAMIENTO Y CREENCIAS RELATIVAS A CONDUCTA ADAPTATIVA EN PACIENTES CON TUMORES ÓSEOS

Revista Mexicana de Análisis de la Conducta, vol. 40, núm. 1, junio, 2014, pp. 81-98

Sociedad Mexicana de Análisis de la Conducta Distrito Federal, México

Disponible en: http://www.redalyc.org/articulo.oa?id=59335810004

Cómo citar el artículo

- Número completo

- Más información del artículo

- Página de la revista en redalyc.org

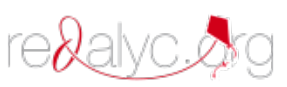

Sistema de Información Científica Red de Revistas Científicas de América Latina, el Caribe, España y Portugal Proyecto académico sin fines de lucro, desarrollado bajo la iniciativa de acceso abierto 


\title{
ESTRÉS, CONDUCTA DE AFRONTAMIENTO Y CREENCIAS RELATIVAS A CONDUCTA ADAPTATIVA EN PACIENTES CON TUMORES ÓSEOS
}

\author{
STRESS, COPING AND ADAPTIVE BEHAVIOR-RELATED BELIEFS \\ IN PATIENTS WITH BONE TUMORS
}

\section{NELLY FLORES-PINEDA, HÉCTOR VELÁZQUEZ-JURADO Y JUAN JOSÉ SÁNCHEZ-SOSA}

\author{
UNIVERSIDAD NACIONAL AUTÓNOMA DE MÉXICO
}

\begin{abstract}
Resumen
Un tumor óseo es una proliferación anormal de células que se origina en el hueso o derivado de cualquiera de las células que lo componen. La literatura especializada los clasifica en dos grandes grupos o estirpes: benignos y malignos o cancerosos. En ambos casos el desajuste conductual, incluyendo sus componentes emocionales, es muy impactante ya que el curso del padecimiento implica eventos generadores de intenso estrés que representa una amenaza seria a diversos aspectos de la vida de los pacientes y su familia inmediata. La medicina conductual ha abordado dichos aspectos asociados con el curso de un padecimiento a fin de mejorar la adaptación de los pacientes. El objetivo del estudio consistió en evaluar las conductas de afrontamiento,

Nelly Flores-Pineda, Héctor Rafael Velázquez-Jurado y Juan José Sánchez-Sosa, División de estudios de posgrado, Avenida Universidad 3004, Facultad de Psicología, Edificio E, cubículo H, C.P. 04510, Copilco Universidad, Coyoacán, México DF.

Correspondencia: Nelly Flores Pineda, Facultad de Psicología, Avenida Universidad 3004, Edificio "E", cubículo H, Col. Copilco Universidad, Código Postal 04510, Del. Coyoacán, México DF. Teléfonos: 56222332 y 0445537336981 . Dirección electrónica: nfloresp@yahoo.com
\end{abstract}


los niveles de estrés percibido y las creencias (Ribes \& Sánchez, 1994) relativas a la propia conducta adaptativa en pacientes con: a) tumores malignos y b) benignos en pacientes de un Instituto Nacional de Salud en México. Participó un total de 82 pacientes atendidos en el Instituto. Los principales hallazgos revelan mayor índice de creencias de conducta adaptativa en los pacientes con tumores malignos y niveles relativamente altos y similares de estrés en ambos grupos. Se concluye que el diagnóstico resulta prácticamente equivalente e intrusivo en sentido conductual incluyendo sus componentes emocionales independientemente de su clasificación, muy probablemente en función de la naturaleza de varios procedimientos médicos. Se comenta la utilidad de los presentes hallazgos para el diseño de intervenciones conductuales en pacientes con cáncer.

Palabras clave: Cáncer, adaptación, medicina conductual, cirugía

\begin{abstract}
Bone tumors consist of abnormal cells proliferation in bones or from bone-derived cells and tissues. The specialized literature classifies them into two broad groups: benign or noncancerous, and malignant or cancerous. In both cases, behavioral maladjustment is severe, since the course of the condition involves life events altered by intense stress, including the perception of a continuous and serious threat to various key aspects of life. Behavioral medicine addresses the issues associated with the course of a disease in order to improve behavioral and emotional adjustment in patients. The purpose of the study was to assess stress levels, self-reported coping behaviors and behavior-related beliefs in patients with either a) malignant or b) benign tumors in a National Institute of Health in Mexico. The main findings revealed higher rates of adaptive behavior beliefs for patients with malignant tumors as well as similar and relatively high levels of stress in both groups. We conclude that the diagnosis of tumor is substantially intrusive for both conditions, mainly because the nature of the medical procedures performed and that behavioral medicine experts could benefit from these findings in order to design appropriate behavioral interventions

Keywords: Cancer, emotional adjustment, behavioral medicine, surgical procedures

Un tumor óseo es una proliferación anormal de células que se origina en el hueso derivado de cualquiera de las células que lo componen. Según la literatura médica, se pueden clasificar en dos grandes grupos: benignos y malignos (Nolla-Solé, 1997, Tecualt, Moreno, \& Amaya, 2008).

Los tumores malignos son cancerosos, tienden a invadir y destruir tejidos, son de rápido crecimiento y tienden a diseminarse a otras partes del cuerpo (metástasis). En contraste, los benignos crecen lentamente, se encuentran delimitados y no hacen
\end{abstract}


metástasis aunque pueden crecer mucho o encontrarse cerca de vasos sanguíneos, nervios u otros órganos que compliquen su tratamiento.

Los pacientes con tumores óseos suelen constituir una población específica, delimitada por la edad y lugar anatómico de incidencia de las lesiones; éstas suelen presentarse con mayor frecuencia en hombres, entre el $75 \%$ y $80 \%$ se diagnostican antes de los 30 años de edad y se localizan principalmente en los huesos largos de las extremidades (Instituto Nacional de Estadística y Geografía [INEGI], 2008; Mendoza, Coutiño, Medina, \& Mora, 2001).

Tanto en la epidemiología internacional como en México, los tumores óseos primarios, si bien presentan una incidencia relativamente baja significan un grave deterioro en el funcionamiento conductual y emocional de los pacientes, no sólo por los síntomas propios de la enfermedad, que incluyen dolor, aumento de volumen e impotencia funcional de las extremidades, sino por las conductas que el ambiente les demanda ejecutar ante el diagnóstico, los tratamientos invasivos y las secuelas que éstos pueden tener. Aunado a lo anterior, en países como México, desde antes de iniciar el proceso de diagnóstico, los pacientes y sus familias suelen enfrentar limitaciones físicas, interpersonales y sociales serias, además de las financieras que entraña la búsqueda de atención médica especializada.

La gravedad de las consecuencias de los tratamientos ortopédicos van desde las complicaciones como la pérdida de funcionalidad de las articulaciones, dolor, hospitalizaciones frecuentes por infecciones, fracturas o cambio de prótesis (Earle, Eiser, \& Grimer, 2005; Flores-Pineda \& Rico, 2007) hasta amputaciones de extremidades pélvicas o torácicas (Covarrubias-Espinoza \& López-Cervantes, 2000; Pacheco, Chávez, Diez, \& Miranda, 2006; Rizo, Sierra. Vásquez, Cano, \& Meneses 2007). En el caso de los tumores óseos malignos, además, se añade el tratamiento oncológico (principalmente quimioterapia) para mejorar el pronóstico de funcionalidad y vida de los pacientes (Barrios, 2008; Covarrubias-Espinoza \& López-Cervantes, 2000; Pacheco et al., 2006).

El curso de este padecimiento en particular, sea benigno o maligno, implica una serie de eventos estresores pues representan una amenaza seria y continua a diversos aspectos de la vida de los pacientes y sus familias.

Las investigaciones respecto al estrés en pacientes con cáncer generalmente se realizan con los que tienen diagnósticos con mayor incidencia y no distinguen a los pacientes con tumores óseos. En general sus hallazgos sugieren que tanto el diagnóstico, la sintomatología y los estrictos regímenes de tratamiento de las enfermedades crónicas como el cáncer, son eventos generadores de estrés tanto agudo como crónico (Andersen \& Wells 2002; Macrodirmitis \& Endler, 2000; Nezu \& Nezu, 1999). Aunado a esto, los tratamientos quirúrgicos constituyen una fuente de estrés intenso, de hecho los pacientes experimentan una alta incidencia de reacciones relacionadas 
con sintomatología ansiosa y depresiva, al tener que enfrentar incomodidad extrema o dolor, y situaciones desconocidas, impredecibles que habitualmente implican riesgos reales sobre su integridad física, adaptación conductual, imagen corporal e incluso su vida si el pronóstico es malo (Bringas et al., 2006; Gavito, Corona, \& Villagrán, 2000; Parsons, Eakin, Bell, Franche, \& Davis, 2008).

En relación a los pacientes con cáncer, es importante no sólo enfocarse en la presencia de estresores sino también en el estrés percibido por su relación con creencias. Esto puede tener mayor importancia que los mismos estresores como lo han descrito varios autores al estudiar por separado tipos de estresores y estrés percibido. Diversos estudios han revelado que el estrés percibido no suele funcionar como un reflejo objetivo del estrés asociado a situaciones concretas (Karademas, Karvelis, \& Argyropoulou, 2007; Kreitler, Peleg, \& Ehrenfeld, 2007).

La literatura de investigación señala que el estrés percibido a partir de la amplia gama de problemas que estos pacientes enfrentan puede estar moderado por las estrategias conductuales que utilizan para solucionarlos (Carver \& Scheier, 2009; Nezu, A. M, Nezu, C. M., Felgoise, McClure, \& Houts, 2003). En el caso de los pacientes con tumores malignos se ha documentado el uso que requieren de recursos conductuales de afrontamiento incluyendo el manejo de creencias de funcionalidad adaptativa para hacer frente al curso de su enfermedad y mejorar el ajuste psicológico a la misma (Andersen \& Wells, 2002; Durá \& Ibáñez, 2000; Gaviria et al., 2007; Nezu et al., 2003; Simpson, 2005; White, Hyde, O`Connor, Naumann, \& Hawkes, 2010).

En el contexto de la Medicina Conductual las creencias de funcionalidad adaptativa se pueden definir como dimensiones compartidas por las acciones de un individuo que funcionan como fundamento de lo que hace. Este fundamento se puede inferir y describir a partir de las propias acciones, y de las consecuencias y circunstancias de sus acciones pasadas y las de otros (Ribes \& Sánchez, 1994, p. 65). De acuerdo con esta propuesta, las creencias pueden describir el sentido que articula a un conjunto de prácticas del paciente en el contexto particular de su padecimiento y de sus circunstancias. En principio, si no se dieran esas prácticas estructuradas de manera particular al afrontar el estrés, la actuación del individuo carecería de sentido. En efecto, los resultados ante los eventos estresantes no sólo resultan de un proceso que involucra la percepción de una persona del estrés y sus esfuerzos de afrontamiento, sino también de sus creencias de funcionalidad adaptativa (Font \& Cardoso, 2009; Folkman 1997; Walco, Conte, Labay, Engel, \& Zeltzer, 2005).

En el caso del paciente con cáncer, diversos estudios señalan que a mayor uso de conductas de evitación, existe mayor presencia de estrés percibido incluso desde antes de la toma de biopsias y continuar, independientemente de que el diagnós- 
tico sea benigno o maligno (Carver \& Scheier, 2009; Witek-Janusek, Gabram, \& Mathews, 2007).

En contraste, el uso de conductas de afrontamiento enfocadas al problema, tales como la reevaluación positiva y la solución de problemas se encuentra asociado con menores niveles de estrés y en general con mayor bienestar informado por los pacientes con cáncer (Andersen \& Wells, 2002; Carver \& Scheier, 2009; Nezu et al., 2003).

Así, la creencia del individuo de poder desempeñar las conductas necesarias que conduzcan a consecuencias positivas creencias de funcionalidad adaptativa (CCAs) puede afectar el funcionamiento del individuo en general y del paciente con cáncer en particular (Luszczynska, Mohamed, \& Schwarzer et al., 2005) ante situaciones demandantes y estresantes que estos padecimientos generan (Bandura, 1997). Así, las creencias de funcionalidad adaptativa pueden facultar a los individuos para emitir conducta instrumental de acuerdo al entorno que la enfermedad representa (Clark, 2008, Godoy-Izquierdo et al., 2007; Kreitler et al., 2007).

De lo anterior surge el interés de la medicina conductual por abordar los aspectos comportamentales y emocionales, incluyendo el papel de creencias de funcionalidad adaptativa, asociados con el curso del padecimiento por las serias repercusiones en el bienestar de los pacientes y sus familias. En virtud de que los pacientes con tumores óseos se enfrentan a una serie de eventos estresantes particulares que los diferencian de otras poblaciones con tumores, se realizó el presente estudio con el objetivo de evaluar a esta población, en cuanto a los aspectos comportamentales que la literatura señala como relevantes para el ajuste o funcionalidad adaptativa ante su padecimiento.

\section{Método}

\section{Participantes}

Participaron 82 pacientes del Instituto Nacional de Rehabilitación, divididos en dos grupos, el primero con diagnóstico de tumores óseos benignos $(n=41)$ y el segundo con tumores óseos malignos $(n=41)$. Los grupos quedaron apareados por edad y género ( 24 hombres, 17 mujeres), con edades oscilando entre 16 y 73 años $(M=33.4, D E=17.76)$, de los cuales 20 se encontraban en el grupo de edad entre 16 y 24 años, 6 tenían entre 25 y 34 años, 4 entre 35 y 44 años, 8 entre 45 y 64 años y solamente 3 entre 65 y 73 años. En cuanto a escolaridad la mitad se distribuyó en quienes contaban con estudios de primaria (18.3\%) y secundaria (34.1\%), alrededor del $30 \%$ con estudios de bachillerato o carrera técnica, el resto (17\%) con estudios de licenciatura y solamente dos sin estudios. 


\section{Medición}

Por las particularidades que caracterizan a los pacientes con tumores óseos, previamente se investigó la validez de una serie de escalas para esta población, mediante la aplicación de las mismas a 200 pacientes con tumores óseos, 148 con tumores benignos y 52 con tumores malignos, con un rango de edad entre 15 y 82 años $(M=$ 34.52 y $D E=16.8$ ).

Escala de Afrontamiento de Moos. Forma B (Moos, Cronkite, Billings, \& Finney, 1986), adaptada, validada y utilizada en población mexicana con padecimientos crónicos (Cortazar-Palapa, Riveros, \& Sánchez-Sosa, 2004; Riveros, Cortazar-Palapa, Alcázar, \& Sánchez-Sosa, 2005; Gutiérrez, 2008). En virtud de su nivel de medida y el tamaño del grupo, en un estudio previo al presente se analizaron los datos de las 200 aplicaciones con la prueba $t$ de Student para muestras independientes sobre cada reactivo del cuestionario a fin de determinar si éstos discriminaban entre los puntajes altos y bajos. De los 30 reactivos originales, 27 lograron discriminar con una probabilidad asociada $p<0.001$. Los reactivos 9,10 y 24, se eliminaron al no mostrar diferencias entre los puntajes altos y bajos. Se calculó el coeficiente alfa de consistencia interna de Cronbach, el cual arrojó un valor de 0.857.

Adicionalmente se determinó la estructura factorial de la escala. Se buscó la mejor opción mediante el criterio de eliminar los reactivos que cargaban en dos o más factores y se procuró que hubiera por lo menos dos reactivos en cada factor, que hubiera congruencia teórica y que conservara el mayor porcentaje posible de varianza explicada. Como resultado se obtuvieron cinco factores que, con 20 reactivos explican el $54.32 \%$ de la varianza. La Tabla 1 . muestra cada factor, el número de reactivos que lo componen, los valores Eigen, los porcentajes de varianza simples y acumulados y el alfa de Cronbach para cada factor. La prueba de esfericidad de Bartlett, mostró un valor aceptable $(\mathrm{Chi}=1039.483, p<.000)$; la prueba $\mathrm{KMO}$ también mostró un valor aceptable $(.794>.5)$.

La Tabla 2 muestra la matriz factorial rotada con la distribución de los reactivos en cada uno de los factores obtenidos y se incluye el alfa para cada factor.

Finalmente, la Tabla 3 muestra los cinco factores obtenidos y sus reactivos, con las siguientes áreas: el primero de Análisis Lógico; el segundo de Búsqueda de Guía y de Apoyo; el tercero de Reevaluación; el cuarto factor de Guía Espiritual (aunque con sólo dos reactivos, mostró un peso factorial importante), finalmente los reactivos del factor cinco, corresponden a conducta de Evitación.

El Análisis Lógico es la estrategia cuyo objetivo es prepararse para enfrentarse con el estresor y sus consecuencias sobre la base de experiencias pasadas e implica la planeación anticipada para enfrentarse con el estresor. La Búsqueda de Guía y apoyo tiene como objetivo buscar información, apoyo y ayuda y prever cursos de acción y 
Tabla 1

Valores Eigen y porcentaje de varianza explicada

\begin{tabular}{ccccc}
\hline Factor & $\begin{array}{c}\text { Núm de reac- } \\
\text { tivos }\end{array}$ & Valor Eigen & \% de varianza & $\begin{array}{c}\text { \% de varianza } \\
\text { acumulada }\end{array}$ \\
\hline 1 & 6 & 4.8951 & 24.475 & 24.475 \\
2 & 3 & 2.062 & 10.309 & 34.784 \\
3 & 5 & 1.479 & 7.394 & 42.178 \\
4 & 2 & 1.227 & 6.136 & 48.314 \\
5 & 3 & 1.202 & 6.012 & 54.327 \\
\hline
\end{tabular}

Nota: Los factores se nombraron en función de los reactivos pertenecientes como 1: Análisis lógico, 2: Búsqueda de guía y apoyo, 3: Reevaluación, 4: Guía espiritual y 5: Evitación.

Tabla 2

Factores obtenidos, con los ítems respectivos y sus cargas factoriales

\begin{tabular}{ccccc}
\hline $\begin{array}{c}\text { Análisis lógico } \\
\text { Ítem /peso f }\end{array}$ & $\begin{array}{c}\text { Búsqueda de } \\
\text { guía y apoyo } \\
\text { Item /peso } f\end{array}$ & $\begin{array}{c}\text { Reevaluación } \\
\text { Ítem /peso } f\end{array}$ & $\begin{array}{c}\text { Guía espiritual } \\
\text { Ítem /peso f }\end{array}$ & $\begin{array}{c}\text { Evitación } \\
\text { Ítem/peso } f\end{array}$ \\
\hline $14 / .788$ & $03 / .692$ & $30 / .681$ & $06 / .862$ & $27 / .744$ \\
$15 / .722$ & $04 / .664$ & $20 / .590$ & $05 / .832$ & $17 / .732$ \\
$13 / .692$ & $02 / .613$ & $29 / .589$ & & $28 / .668$ \\
$12 / .539$ & & $25 / .473$ & & $22 / .477$ \\
$07 / .523$ & & $21 / .450$ & & \\
$11 / .474$ & & & & \\
\hline Alfa Factor 1 & Alfa Factor 2 & Alfa Factor 3 & Alfa Factor 4 & Alfa Factor 5 \\
.775 & .671 & .591 & .804 & .621 \\
\hline
\end{tabular}

sus posibles resultados ante el estresor. La Reevaluación cubre las estrategias mediante las cuales un individuo acepta las características básicas de la situación, pero las re- 
conceptualiza o reestructura para fomentar conductas adaptativas. La Evitación es la estrategia para evitar pecibir o conceptualizar el problema o estresor, para negarlo o minimizar el tamaño o gravedad de la crisis (Moos \& Schaefer, 1993). Se consideró nombrar al factor Guía Espiritual a los dos reactivos que señalaban la búsqueda de guía y apoyo en base creencias espirituales (Folkman, 1997).

Tabla 3

Reactivos pertenecientes a cada factor

\begin{tabular}{|c|c|}
\hline Factor & Reactivos que constituyen al factor \\
\hline 1. Análisis lógico & $\begin{array}{l}\text { 14. Pienso en varias opciones para manejar el problema. } \\
\text { 15. Pienso en cómo resolví problemas parecidos anteriormente. } \\
\text { 13. Pienso en un plan de acción y lo echo a andar. } \\
\text { 12. Hago otras cosas para no pensar en el problema. } \\
\text { 07. Me preparo por si pasa lo peor. } \\
\text { 11. Trato de verle el lado bueno al problema. }\end{array}$ \\
\hline $\begin{array}{l}\text { 2. Búsqueda de } \\
\text { guía y apoyo }\end{array}$ & $\begin{array}{l}\text { 03. Hablo con un(a) amigo(a) sobre el problema. } \\
\text { 04. Hablo con un profesionista (médico, abogado, cura, etc.) } \\
\text { sobre el problema. } \\
\text { 02. Hablo con mi pareja o con otros parientes sobre el problema. }\end{array}$ \\
\hline 3. Reevaluación & $\begin{array}{l}\text { 30. Trato de sacarle provecho al problema. } \\
\text { 20. Trato de no actuar precipitadamente. } \\
\text { 29. Busco la ayuda de personas o grupos que hubieran tenido } \\
\text { experiencias similares. } \\
\text { 25. Trato de que en un futuro las cosas sean diferentes. } \\
\text { 21. Me digo cosas a mis mismo(a) para sentirme mejor. }\end{array}$ \\
\hline 4. Guía espiritual & $\begin{array}{l}\text { 06. Rezo para darme valor. } \\
\text { 05. Le pido a Dios que me guíe. }\end{array}$ \\
\hline $\begin{array}{l}\text { 5. Evitación } \\
\text { cognitiva }\end{array}$ & $\begin{array}{l}\text { 27. Como yo no puedo hacer nada, lo acepto. } \\
\text { 17. Tomo las cosas como vienen. } \\
\text { 28. Trato de no sentir nada. } \\
\text { 22. Trato de olvidarme por un tiempo del problema. }\end{array}$ \\
\hline
\end{tabular}

Escala de Percepción del Estrés (Cohen, Kamarck, \& Mermelstein, 1983). Originalmente consta de 14 reactivos, por haberse diseñado para personas con al menos 
estudios de secundaria, se instrumentó un estudio para validar la escala con las 200 aplicaciones ya mencionadas. Inicialmente se excluyó el reactivo 12 por no discriminar en la prueba t de Student entre puntajes bajos y altos. Dado que el coeficiente obtenido se consideró bajo, se eliminaron aquellos reactivos que el análisis indicó que elevarían el coeficiente. Así, se excluyeron los reactivos 5, 7 y 10 con lo que la escala conservó diez elementos desde los cuales se estableció su consistencia interna mediante el coeficiente alfa de Cronbach, la cual arrojó un valor de 0.791.

Escala General de Autoeficacia, de Schwarzer y Jerusalem (1995). La traducción al español (Baessler \& Schwarzer, 1996), se ha administrado en muestras de diversas nacionalidades: españoles, peruanos y mexicanos entre otros (Grimaldo, 2005; San Juan et al., 2000; Padilla et al., 2006). Para la presente investigación, se calculó la consistencia interna de la escala la cual arrojó un valor Alfa de Cronbach de 0.862 con los diez reactivos originales.

\section{Procedimiento}

A los pacientes se les abordaba tanto en la sala de espera como en el área de hospitalización, se les describía en términos generales el propósito del estudio y se les invitaba a participar contestando los instrumentos en un formato sencillo tipo entrevista. El aplicador entregaba un juego de instrumentos al paciente para que fuera siguiendo con la vista las preguntas y opciones de respuesta, mientras el aplicador las leía en voz alta y anotaba la respuesta elegida.

\section{Resultados}

El análisis del apareamiento en función de las variables sociodemográficas registradas (edad, sexo y escolaridad) como para garantizar la comparabilidad de los sujetos, en efecto, no arrojó diferencias estadísticamente significativas en las variables de sexo y escolaridad. En principio, por tanto, las diferencias son atribuibles al tipo de tumor. Para el caso de las creencias de funcionalidad adaptativa y las conductas de afrontamiento análisis lógico y búsqueda de guía, los resultados muestran que fue el grupo de menor edad el que puntuó más bajo en estas variables. Para examinar las diferencias en los resultados de las escalas de creencias de funcionalidad adaptativa, percepción de estrés y afrontamiento, en función del tipo de tumor (benigno o maligno), se compararon las medias por medio de la prueba t de Student para muestras independientes (Tabla 4). 
Tabla 4

Comparación de Medias por grupo, N = 82 (t de Student)

\begin{tabular}{cccc}
\hline Variable & x benignos & x malignos & $p$ \\
\hline $\begin{array}{c}\text { Creencias de Conducta } \\
\text { Adaptativa } \\
\text { Estrés }\end{array}$ & 30.59 & 33.37 & 0.030 \\
Análisis lógico & 17.07 & 17.44 & 0.825 \\
Búsqueda de guía y apoyo & 19.82 & 20.46 & 0.624 \\
Reevaluación & 8.7 & 9.39 & 0.379 \\
Guía espiritual & 19.22 & 14.80 & 0.700 \\
Evitación & 7.53 & 7.95 & 0.455 \\
& 11.43 & 13.02 & 0.080 \\
\hline
\end{tabular}

Los resultados del análisis muestran que existen diferencias significativas en la variable creencias de funcionalidad adaptativa, son los pacientes con tumores malignos los que obtuvieron puntajes más altos. El resto de las variables no mostró diferencias importantes entre los grupos.

Posteriormente se diseñaron gráficas de «caja y bigote» (box \& whiskers) para determinar la forma en que se distribuyen los datos de acuerdo con el grupo de pertenencia.

En la Figura 1 se muestran los puntajes en creencias de funcionalidad adaptativa, la tendencia de éstos en el grupo de malignos señala hacia los puntajes más altos. No hubieron valores extremos en ningún grupo.

La Figura 2 muestra la distribución de los puntajes correspondientes a los niveles de estrés percibido para cada grupo. A pesar de que el cuartil 1, la mediana y el cuartil 3 se observan agrupados de manera similar en cada grupo, se observa que el grupo de pacientes con diagnóstico de tumores benignos arrojó puntajes más altos. Tampoco hubieron valores extremos en ningún grupo. 


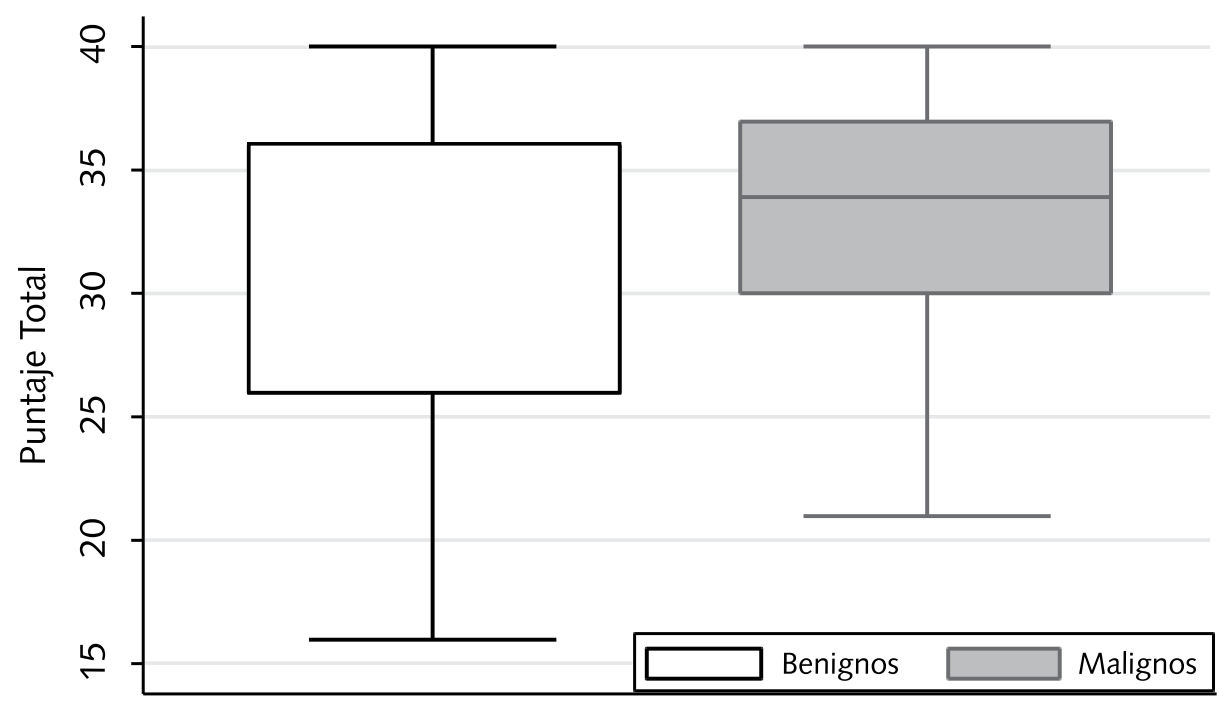

Figura 1. Nivel de creencias de conducta adaptativa por estirpe

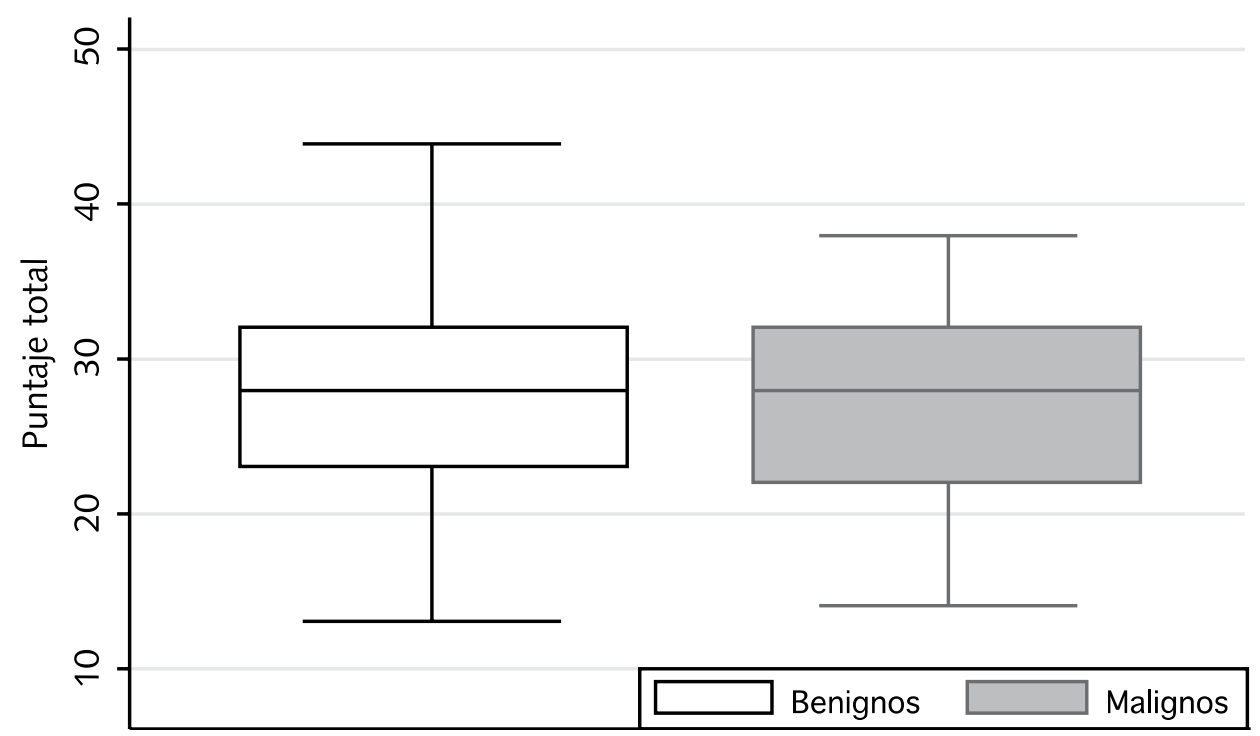

Figura 2. Nivel de estrés por estirpe 
Para analizar la relación de las variables comportamentales entre sí y con algunos datos sociodemográficos se utilizó el coeficiente de correlación de Pearson. Se calcularon correlaciones entre los puntajes totales de las Escalas de Estrés, de creencias de funcionalidad adaptativa y de cada uno de los factores de la Escala de Afrontamiento. Se obtuvo una correlación negativa significativa entre creencias de funcionalidad adaptativa y estrés $(r=-.428, p<.001)$, y los factores de afrontamiento análisis lógico $(r=.501, p<.001)$, búsqueda de guía $(r=.434, p=.004)$ y reevaluación $(r=.434, p<$ .001). A su vez, el factor análisis lógico correlacionó significativamente con el factor búsqueda de guía $(r=.451, p<.001)$, con el factor reevaluación $(r=.536, p<.001)$ y el factor guía espiritual $(r=.436, p<.001)$, así como con el estrés percibido $(r=-.289$, $\mathrm{p}<.001)$. Otras correlaciones significativas se encontraron entre el factor búsqueda de guía y los factores reevaluación $(r=.388, p<.001)$ y guía espiritual $(r=.410, p$ $<.001)$. Finalmente reevaluación y guía espiritual correlacionaron significativamente entre sí $(r=.312, p<.001)$, así como reevaluación y evitación $(r=.289, p<.001)$.

\section{Discusión}

El objetivo del presente estudio consistió en evaluar en pacientes con tumores óseos, algunas de las variables psicológicas más importantes que, de acuerdo con la literatura de investigación se asocian con creencias de funcionalidad adaptativa y conductas que dichos pacientes requieren para un adecuado ajuste a su padecimiento (Andersen \& Wells, 2002; Godoy-Izquierdo et al., 2007; Nezu et al., 2003). Asimismo se buscó examinar en esta población, las propiedades psicométricas de las escalas utilizadas, toda vez que, a pesar de haber sido adaptadas a población mexicana, no se habían empleado en el tipo de pacientes participantes.

El interés por realizar este estudio surge de la demanda de atención integral por parte de los pacientes hospitalizados en un servicio de tumores óseos, donde expertos en medicina conductual buscan intervenir junto con los especialistas en ortopedia en los procesos de diagnóstico y tratamiento. Así el objetivo incluye proveer el contexto profesional que facilite los cambios emocionales, creencias de funcionalidad adaptativa y conductuales hacia una adaptación que mejore la calidad de vida de los pacientes (Flores-Pineda, 2008).

Los pacientes con tumores óseos malignos refieren asumirse más capaces de desempeñar conductas adaptativas que los que tienen un diagnóstico benigno y creen tener la capacidad de hacer frente al diagnóstico y tratamiento de su enfermedad. Estos pacientes parecen inferir que pueden manejar los desafíos de la enfermedad, a partir de sus conductas de afrontamiento exitoso ante problemas anteriores. Se ha señalado que las personas con creencias de funcionalidad adaptativa eligen desempeñar tareas más desafiantes, colocándose metas y objetivos más altos. En el caso 
de los pacientes con tumores malignos, estos desafíos estarían en función de las situaciones estresantes que tienen que aceptar como parte de su realidad, así como las implicaciones de la enfermedad y la posibilidad de un curso reversible (Bandura, 1997; Schwarzer et al., 2005).

En cuanto al estrés percibido, ambos grupos de pacientes refirieron niveles relativamente similares, en relativo contraste con lo esperado. A pesar de que no se observaron diferencias estadísticamente significativas entre los grupos, al observar la distribución de los datos, los pacientes con diagnóstico benigno muestran los puntajes más altos en este rubro. Estos hallazgos podrían explicarse en vista de que todos los pacientes al ingresar al servicio de tumores óseos tienen que pasar a un protocolo de estudio. De tal manera que en ese punto, aún sin un diagnóstico confirmado, los pacientes con tumores benignos recorren el mismo proceso de diagnóstico que los malignos, con todas sus características e implicaciones de reacciones emocionales. El traslado a la institución, los gastos económicos, los estudios a realizarse, el estar hospitalizados, la biopsia en quirófano y el tiempo de espera de resultados son factores importantes que comparten por igual con los pacientes con tumores malignos y que, desde antes del tratamiento podrían estar elevando su estrés, como se ha documentado en pacientes con otro tipo de tumor (Bringas et al., 2006; Flores-Pineda, 2008; Gavito, Corona, \& Villagrán, 2000; Rico et al., 2007). También es de notarse que ciertos tipos de tumores benignos llegan a ser agresivos, extendiéndose y envolviendo tejido sano, con el consecuente dolor o incomodidad extrema; otros reaparecen después de la cirugía, lo cual demanda nuevos tratamientos frecuentemente igual de invasivos. Algunos pacientes se enfrentan a tratamiento por infecciones, cambio de prótesis o dolor, en ocasiones llegan a perder la funcionalidad de las articulaciones y los casos más graves, requerir amputación (Pacheco et al., 2006; Flores-Pineda \& Rico, 2007). Los anteriores constituyen estresores importante en la vida de dichos pacientes. Aunque no es común que lleguen a malignizarse, las recomendaciones médicas incluyen la vigilancia del tumor incluso por años, esta conducta de cuidado, que implica un monitoreo constante, también estaría elevando los niveles de estrés en los pacientes a pesar del diagnóstico de benignidad.

Los resultados del presente estudio extienden hallazgos previos en los que el estrés se encuentra relacionado negativamente con las Creencias de Conducta adaptativa, comprendidas usualmente en otras tradiciones en el término autoeficacia (Clark, 2008; Schwarzer et al., 2005). Así, mientras mayores son las creencias de funcionalidad adaptativa del individuo sobre sí mismo para hacer frente a las demandas de las situaciones, el estrés es menor. Esto podría explicarse mediante dos posibles mecanismos. El más común incluiría considerar que la percepción de competencia que entrañan las creencias de funcionalidad adaptativa es relativamente independiente de si, en efecto, se tengan las habilidades o no, e independiente de que los resultados esperados se obtengan. En este caso, a medida 
que la creencia de conducta adaptativa incrementa sería suficiente para percibir menor estrés (Bandura, 1997; Clark, 2008; Godoy-Izquierdo et al., 2007; Kreitler et al., 2007). Incluso algunas investigaciones refieren que los pacientes con tumores malignos emplean las creencias de funcionalidad adaptativa como estrategia de afrontamiento al estrés (Clark, 2008; Schwarzer et al., 2005). En el otro sentido, mayor estrés percibido podría indicar que la persona considera las situaciones tan amenazantes que sobrepasan sus recursos conceptuales, de creencias de funcionalidad adaptativa y conductuales. Así, los pacientes no sabrían cómo actuar para resolver los problemas que se les presentan, lo que podría desafiar la creencia de poder hacer frente a la situación.

Un hallazgo adicional indica que a menor edad los puntajes creencias de funcionalidad adaptativa y las estrategias de afrontamiento referentes al análisis lógico y a la búsqueda de guía y apoyo, son más bajos. Al parecer la experiencia asociada con la edad facilita en las personas un cambio conceptual sobre sus capacidades instrumentales para resolver problemas. Estos hallazgos concuerdan con hallazgos de otros estudios que encontraron que la efectividad del afrontamiento se encuentra relacionado con los cambios que se dan con la edad sobre las creencias personales y sobre el estrés percibido (Trouillet, Gana, Lourel, \& Fort, 2009; Schwazer et al., 2005).

Estas posibilidades reflejan o implican la importancia de sugerir intervenciones conductuales en los pacientes más jóvenes que, coincidentemente son el grupo de edad en que se presentan la mayoría de los padecimientos tumorales en huesos (Covarrubias-Espinoza \& López-Cervantes, 2000; INR, 2007). Por otro lado, hay que considerar que los pacientes menores de 18 años de edad, se encuentran sujetos a las decisiones de sus padres sobre aceptar o no los tratamientos. En estos casos la sensación de falta de control podría ser mayor, primero por estar enfermo, segundo por no poder suponer control personal y tercero, por el carácter invasivo del tratmiento mismo.

La asociación entre los factores de la escala de afrontamiento, análisis lógico, búsqueda de guía y apoyo y revaluación se refieren al tipo de afrontamiento dirigido al problema (Gaviria et al., 2007; Nezu et al., 2003). La correlación negativa encontrada entre análisis lógico y estrés, brinda orientación clínica en términos de las intervenciones conductuales con este tipo de pacientes. Estos hallazgos apoyan el tratamiento psicológico recomendado para pacientes con cáncer (tumores malignos), el cual considera la explicación razonada del estrés y su relación con este padecimiento, antes de introducir cambios conductuales en los pacientes para el manejo del estrés por medio de intervenciones de solución de problemas (Nezu et al., 2003; Clark, 2008). Se sugiere en este caso en particular, buscar de manera rutinaria en los pacientes hacer un abordaje comportamental y emocional para apoyar a pacientes con lesiones tanto benignas como malignas. 
Aunque en el presente estudio se formaron dos grupos de pacientes (con tumores óseos benignos y malignos) con características comparables; hay que considerar que dentro de esta gran división, existen más de cuarenta tipos de tumores con características particulares condicionadas por el comportamiento biológico de las células de origen. Este comportamiento se refleja en su sintomatología más allá del dolor, aumento de volumen tumoral y limitación funcional, incluyendo crecimiento acelerado del tumor, riesgo de fractura, involucramiento de tejidos blandos y ataque al bienestar general (Tecualt, Moreno, \& Amaya, 2008). Para estudios futuros convendría establecer, en lo posible, comparaciones más finas entre estas categorías.

\section{Referencias}

Andersen, B. \& Wells, S. (2002). Cancer. En: A. Christensen \& M. Antoni. (Eds.), Chronic Physical Disorders. Behavioral Medicine`s Perspective. (pp. 112-136) Oxford, UK: Blackwell Publishers Ltd.

Bandura, A. (1997). Self-efficacy: The exercise of control. New York: Freeman.

Barrios, C. (2008). Tumores Óseos. Unidad de Traumatología y Cirugía Ortopédica. Facultad de Medicina. Universidad de Valencia. Recuperado de http://centros. uv.es/web/departamentos/D40/data/informacion/E125/PDF746.pdf

Bringas, S., Krikorian, A., Hernando, F., Rodríguez, O., Jarabo, J. R., Fernández, E., ... Balibrea, J. L. (2006). Protocolo de intervención psicosocial en pacientes oncológicos sometidos a cirugía torácica. Psicooncología, 3, 2-3.

Carver, C. S., \& Scheier, M. F. (2009). Coping processes and adjustment to chronic illness. En A. J. Christensen \& M. H. Antoni (Eds.), Chronic physical disorders: Behavioral medicine's perspective (pp. 47-68). Oxford, UK: Blackwell.

Cohen, S., Kamarck, T., \& Mermelstein, R. (1983). A global measure of perceived stress. Journal of Health and Social Behavior, 24(4), 385-396

Cortázar-Palapa, J., Riveros, R.A., \& Sánchez-Sosa, J. J. (2004, septiembre). Herramientas para la toma de decisiones en terapia cognitivo-conductual en escenarios médicos: el uso del sistema de autorregistro. Ponencia presentada en el XII Congreso Mexicano de Psicología, Guanajuato, México.

Covarrubias-Espinoza, G., \& López-Cervantes, G. (2000). Osteosarcoma. Quimioterapia pre y postoperatoria. Informe de 10 casos. Hospital Infantil de Estado de Sonora. Revista del Instituto Nacional de Cancerología, 46 (4), 251-254.

Durá, E., \& Ibáñez, E. (2000). Psicología Oncológica: perspectivas futuras de investigación e intervención profesional. Psicología, Saúde \& Doencas, 1 (1), 27-43.

Earle, E.A., Eiser, C., \& Grimer, R. (2005). "He never liked sport anyway"- Mother's views of young people coping with a bone tumor in the lower limb. Sarcoma, 9 (1/2), 7- 13. 
Fierro, V. F. J. (2008). Frecuencia de tumores óseos primarios observados en biospias de hueso obtenidas en dos centros clínicos de la ciudad de Guadalajara, Jalisco (Tesis de Maestría). Universidad de Colima.

Flores-Pineda. N., \& Rico M. G. (noviembre, 2007). Calidad de Vida en Pacientes con Artrodesis de Rodilla. Trabajo presentado en el XIX Congreso Nacional de la Asociación Mexicana de Ortopedia y Traumatología, Mérida, México.

Flores-Pineda, N. (2008). Intervención psicológica de pacientes programados a cirugía de artrodesis de rodilla (Tesis de Maestría). Facultad de Psicología, Universidad Nacional Autónoma de México.

Folkman, S. (1997). Positive psychological states and coping with severe stress. Social Science \& Medicine, 45(8), 1207-1221.

Font, A., \& Cardoso, A. (2009). Afrontamiento en cáncer de mama: Pensamientos, conductas y reacciones emocionales. Psicooncología, 6(1), 27-42.

Gaviria, A. M., Vinaccia, S., Riveros, M., \& Quiceno, J. (2007). Calidad de vida relacionada con la salud, afrontamiento del estrés y emociones negativas en pacientes con cáncer en tratamiento quimioterapéutico. Psicología desde el Caribe. Universidad del Norte. 20, 50-75.

Gavito, M. C., Corona, M. A., \& Villagrán, M. E. (2000). La información anestésica quirúrgica: su efecto sobre la ansiedad y el dolor de los pacientes toracotomizados. Revista del Instituto Nacional de Enfermedades Respiratorias de México, 13(3), 153-156.

Griamaldo, M. M. P. (2005). Propiedades Psicométricas de la escala de Autoeficacia General de Baessler \& Schwrzer. Cultura. Revista de la Asociación de docentes de la Universidad de San Martín de Porres. Lima, Perú, 23(19), 213-229.

Godoy Izquierdo, D., Godoy García, J. F., López-Chicheri García, I., Martínez Delgado, A., Gutiérrez Jiménez, S., \& Vázquez Vázquez, L. (2008). Propiedades psicométricas de la escala de autoeficacia para el afrontamiento del estrés (EAEAE). Psicothema, 20(1), 155-165.

Gutiérrez, M. I. (2008). Estilos de afrontamiento en estudiantes universitarios con Síndrome de Intestino Irritable (Tesis de Licenciatura). Facultad de Psicología, Universidad Nacional Autónoma de México.

Instituto Nacional de Estadística, Geografía e Informática (2008). Estadísticas a propósito del día mundial contra el cáncer. Datos Nacionales. Recuperado de http:// www.inegi.gob.mx

Instituto Nacional de Rehabilitación. Portal Discapacinet. (s.f.) Discapacidades, Prevención y Rehabilitación. Tumores Óseos. Recuperado de http://e-mexico.gob. $\mathrm{mx} / \mathrm{wb} 2 / \mathrm{eMex}$ eMex_tumores_oseos

Karademas, E.C., Karvelis S., \& Argyropoulou, K. (2007). Short communication: Stress related predictors of optimism in breast cancer survivors Stress and Health, 23, 161-168. 
Kreitler, S., Peleg, D., \& Ehrenfeld, M. (2007). Stress, self-efficacy and quality of life in cancer patients. PsychoOncology, 16(4), 329-341.

Martínez, A. R., Arizmendi, I.S.A., Flores, V.F., Barra M.R., Díaz R.,L., \& De la Garza, J. M. (2004). Tratamiento del osteosarcoma: experiencia de 10 años en el Hospital General de México. Gamo, 3(2), 38-40.

Mendoza C., Coutiño B., Medina A., \& Mora , I. (2001). Programa de rehabilitación pre y posoperatorio para pacientes pediátricos con tumores óseos malignos primarios en extremidades, manejados con cirugías de salvamento. Revista Mexicana de Medicina Física y Rehabilitación, 13, 44-49.

Moos, R., Cronkite, R.C., Billings, A. G., \& Finney, J. (1986). The health and daily living form manual. Social Ecology Laboratory, Department of Psychiatry and Behavioral Science. Palo Alto, CA: Veterans Administration and Stanford University Medical Center.

Moos, R. H., \& Schaefer, J. A. (1993). Coping resources and processes: Current concepts and measures. En L. Goldberg \& S. Breznitz (Eds.) Handbook of stress: Theoretical and clinical aspects. (pp. 234-257) New York, N.Y., Free Press.

Nezu, A. M., Nezu, C. M., Felgoise, S. H., McClure, K. S., \& Houts, P. S. (2003). Project Genesis: assessing the efficacy of problem-solving therapy for distressed adult cancer patients. Journal of consulting and clinical psychology, 71(6), 1036.

Nolla-Solé, J.M. (1997). Enfermedades Óseas, Masson: España.

Pacheco, M.R., Chávez, D., Diez, M.P., \& Miranda, A. (2006). Control de casos de pacientes amputados por tumor en el Instituto Nacional de Rehabilitación. Seguimiento de 6 años. Revista Mexicana de Medicina Física y Rehabilitación, 18, 7-10.

Padilla, J.L., Acosta, B., Guevara, M., Gómez, J., \& González, A. (2006). Propiedades psicométricas de la versión española de la Escala de Autoeficacia General Aplicada en México y España. Revista Mexicana de Psicología, 23(2), 245-252.

Parsons, J., Eakin, J.M., Bell, R.S., Franche, R., \& Davis, A.M. (2008). "So, are you back to work yet?" Re-conceptualizing 'work' and 'return to work' in the context of primary bone cancer. Social Science and Medicine, 67, 1826-1836.

Ribes, E., \& Sánchez, S. (1994). Conducta, juegos de lenguaje y criterios de validación del conocimiento. Acta Comportamentalia, 2, 57-86.

Rico M.G., Delgado-Cedillo, E., Estrada E., González R., Flores-Pineda N. (2007) Los dilemas en tumores óseos. Acta Ortopédica Mexicana, 21 (6).

Riveros, A., Cortazar-Palapa, J., Alcazar, L., \& Sánchez-Sosa, J. J. (2005). Intervención cognitivo-conductual en pacientes diabéticos e hipertensos esenciales. International Journal of Clinical and Health Psychology, 5(3), 445-462.

Rizo P., Sierra M.I., Vásquez G., Cano, M., Meneses, A., \& Mohar, A. (2007). Registro Hospitalario de Cáncer. Compendio de cáncer 2000-2004. Cancerología, 2, 203-287. 
Sanjuán Suárez, P., Pérez García, A. M., \& Bermúdez Moreno, J. (2000). Escala de autoeficacia general: datos psicométricos de la adaptación para población española. Psicothema, 12 (Suplemento), 509-513.

Schwarzer, R., \& Baessler, J. (1996). Evaluación de la autoeficacia: adaptación española de la Escala de Autoeficacia General. Ansiedad y estrés, 2(1), 1-8.

Schwarzer R., \& Jerusalem W. (1995). Generalized self-efficacy scale. En J. Weinman, J. Wright, \& M. Johnston. Measures in health psychology: A user's portfolio. Causal and control beliefs (pp. 35-37). Windsor, England: WFER-WELSON.

Simpson, P. (2005). Hong Kong Families and Breast Cancer: Beliefs and Adaptation Strategies. Psycho-Oncology, 14(8), 671-683.

Schwarzer, R., Boehmer, S., Luszczynska, A., Mohamed, N. E., \& Knoll, N. (2005). Dispositional self-efficacy as a personal resource factor in coping after surgery. Personality and Individual Differences, 39(4), 807-818.

Tecualt, G. R., Moreno H.L.F., \& Amaya, Z.R.A. (2008). Clasificación de los tumores óseos. Ortho-tips, 4(2), 96-102.

Walco, G. A., Conte, P. M., Labay, L. E., Engel, R., \& Zeltzer, L. K. (2005). Procedural distress in children with cancer: Self-report, behavioral observations, and physiological parameters. The Clinical Journal of Pain, 21(6), 484-490.

White, K. M., Hyde, M. K., O'Connor, E. L., Naumann, L., \& Hawkes, A. L. (2010). Testing a belief-based intervention encouraging sun-safety among adolescents in a high risk area. Preventive Medicine: An International Journal Devoted to Practice and Theory, 51(3-4), 325-328.

Witek-Janusek, L., Gabram, S., \& Mathews H. (2007). Psychologic stress, reduced NK cell activity, and cytokine dysregulation in women experiencing diagnostic breast biopsy. Psychoneuroendocrinology, 32 (1), 22-35. 

